\title{
openheart The diagnostic and prognostic value of coronary CT angiography in asymptomatic high-risk patients: a cohort study
}

\author{
Fabian Plank, ${ }^{1}$ Guy Friedrich, ${ }^{2}$ Wolfgang Dichtl, ${ }^{2}$ Andrea Klauser, ${ }^{1}$ \\ Werner Jaschke, ${ }^{1}$ Wolfgang-Michael Franz, ${ }^{2}$ Gudrun Feuchtner ${ }^{1}$
}

To cite: Plank F, Friedrich G, Dichtl W, et al. The diagnostic and prognostic value of coronary CT angiography in asymptomatic high-risk patients: a cohort study. Open Heart 2014;1: e000096. doi:10.1136/ openhrt-2014-000096

Received 26 February 2014 Revised 23 May 2014 Accepted 15 July 2014

\section{CrossMark}

\footnotetext{
${ }^{1}$ Department of Radiology, Innsbruck Medical University, Innsbruck, Austria

${ }^{2}$ Department of Cardiology, Innsbruck Medical University, Innsbruck, Austria
}

Correspondence to Dr Gudrun Feuchtner; Gudrun.Feuchtner@i-med. ac.at

\section{ABSTRACT}

Objective: To prospectively assess the value of coronary CT angiography (CTA) in asymptomatic patients with high 'a priori' risk of coronary artery disease (CAD).

Methods: 711 consecutive asymptomatic patients (61.8 years; $40.1 \%$ female) with high 'a priori' risk of $\mathrm{CAD}$ were prospectively examined with a coronary calcium score (CCS) and CTA. Coronary arteries were evaluated for atherosclerotic plaque (non-calcified and calcified) and stenosis (mild $<50 \%$, intermediate $50-70 \%$ or high-grade $>70 \%$ ). Coronary Segment Involvement Score (SIS, total number of segments with plaque) and nc (non-calcified) SIS were calculated. Primary end points were major adverse cardiac events (ST-elevation $\mathrm{MI}$, non-ST-elevation MI and cardiac death); secondary end points were coronary revascularisation and $>50 \%$ stenosis by invasive angiography.

Results: Of 711 patients, $28.3 \%$ were negative for $C A D$ and $71.7 \%$ positive (CAD+) by CTA $(15.6 \%$ had plaques without stenosis, $23.9 \%$ mild, $10.7 \%$ intermediate and $21.5 \%$ high-grade stenosis). CCS zero prevalence was $306(43 \%)$, out of those 100 (32.7\%) had non-calcified plaque only. Mean follow-up period was 2.65 years. MACE rate was $0 \%$ in CAD negative and higher (1.2\%) in CAD positive by CTA. Coronary revascularisation rate was $5.5 \%$. Patients with SIS $\geq 5$ had an HR of $6.5(95 \% \mathrm{Cl}$ 1.6 to $25.8, p<0.013)$ for MACE, patients with ncSIS $\geq 1$ had an HR of $2.4(95 \% \mathrm{Cl} 1.2$ to $4.6, \mathrm{p}<0.01)$ for secondary end point. The sensitivity of CTA for stenosis $>50 \%$ compared with invasive angiography was $92.9 \%$ (95\% Cl 83.0\% to $98.1 \%)$. Negative predictive value of CTA was $99.4 \%$ (95\% Cl $98.3 \%$ to $99.8 \%$ ) for combined end points.

Conclusions: CAD prevalence by CTA in asymptomatic high-risk patients is high. CCS zero does not exclude CAD. CTA is highly accurate to exclude CAD. Total coronary plaque burden and nc plaques, even if only one segment is involved, are associated with an increased risk of adverse outcome.

\section{INTRODUCTION}

Myocardial infarction (MI) in previously asymptomatic patients with subclinical

\section{KEY MESSAGES}

What is already known about this subject?

- Asymptomatic patients without chest pain symptoms but a high coronary risk profile carry a higher risk of myocardial infarct. Currently, CT Calcium Scoring (CCS) is recommended for risk stratification in this cohort.

What does this study add?

- Coronary CT-Angiography adds values over CCS in terms of providing quantification of total and non-calcifying coronary plaque burden (SIS and ncSIS score) and stenosis graduation (mild, intermediate and severe). Our study shows that beyond coronary calcium, non-calcifying plaque burden as well predicts outcome.

How might this impact on clinical practice?

- Coronary CT-Angiography should be considered in asymptomatic patients with high life-time risk of $\mathrm{CAD}$ as more accurate screening tool for CAD due to its ability to detect non-calcifying plaques and to quantify plaque burden (SIS and ncSIS score), as well as coronary stenosis severity graduation.

coronary artery disease (CAD), who remain undetected with conventional screening methods, is linked with high morbidity, mortality and socioeconomic burden.

Further, asymptomatic 'silent' myocardial ischaemia increases the likelihood of future coronary events, particularly in healthy males. ${ }^{1-3}$

Coronary calcium scoring (CCS) using CT is recommended in asymptomatic patients with low or intermediate risk of CAD for detection of calcifying plaque. ${ }^{4}$ Screening of asymptomatic patients with intermediateto-high risk of CAD by coronary CT angiography (CTA) is currently under debate and was graded as an uncertain but potentially useful clinical application according to 2010 ACCF/SCCT/ACR guidelines. ${ }^{5}$ 
CTA has the unique advantage over CCS of detecting non-calcifying plaques in addition to calcifying lesions, ${ }^{6}$ thus allowing for direct visualisation of early atherosclerosis stages such as lipid and fibrous atheroma, which are risk factors for future coronary events. ${ }^{7}$ Long-term studies report of an increased risk of adverse outcome associated with vulnerable fibroatheroma, whereas calcifying lesions tend to remain rather stable. ${ }^{8}$

Studies investigating the accuracy, outcome and, thus, the diagnostic benefit of coronary CTA (CCTA) in asymptomatic patients are scarce. ${ }^{9-12}$

Higher radiation exposure of $12 \mathrm{mSv}$ using 64-slice $\mathrm{CTA}^{13}$ was an issue of concern for using CTA as a widespread screening tool in asymptomatic patients. A variety of recently introduced new CT-technologies such as highpitch CTA, prospective ECG-triggering and iterative reconstruction reduce the radiation dose to $1 \mathrm{mSv}$ or even less, thus equalising the radiation exposure of CCS and CTA. ${ }^{14} 15$

Therefore, the purpose of this study was to assess the value of CCTA in an asymptomatic patient cohort with high 'a priori' risk of CAD based on risk profile and conventional tests and to identify risk factors for adverse outcome (major adverse cardiac events (MACE) and coronary revascularisation).

\section{METHODS}

\section{Study design}

Seven hundred and eleven asymptomatic, consecutive patients referred to CCTA between 2005 and 2012 were included into this prospective. IRB approved study.

Inclusion criteria were: asymptomatic patients with low, intermediate or high Framingham risk profile based on conventional risk factors. Blood tests were performed within a maximum of 14 days prior to CT and definitions were made according to the most recent ESC guidelines: arterial hypertension (systolic blood pressure (SBP) $>140 \mathrm{bpm}$ or diastolic BP $>90 \mathrm{bpm}$ ), dyslipidaemia (total cholesterol $>200 \mathrm{mg} / \mathrm{dL}$ or high-density lipoprotein $<40 \mathrm{mg} / \mathrm{dL}$ ), family history (MI or sudden cardiac death in an immediate male relative $<55$ years or female $<65$ years), smoker (current smoker or those who quit in the past 6 months) and diabetes. ${ }^{16} 17$

A preceding non-invasive ECG-treadmill stress test was scheduled. The patients were included if the test was inconclusive (eg, low physical performance), not feasible due to comorbidity or incompliance, not specific pathological (eg, premature ventricular contraction, arterial blood pressure increase, arrhythmia, etc) or borderline or mild positive for myocardial ischaemia (ST-segment depression of $1-2 \mathrm{mV}) .{ }^{18}$

Patients were finally included if they had a high 'a priori' risk of CAD defined as: either a (1) high-" lifetime' cardiovascular risk estimated by Berry et $a l^{19}$ including patients with diabetes or (2) low lifetime risk ${ }^{19}$ but a borderline or non-specific mild pathological ECG-treadmill stress test result (see criteria above)
After a minimum of 1 year up to 8 years, follow-up was performed via phone call and examining the cardiologist's or hospitalisation chart results of the patient. Our centre is the only invasive coronary angiography (CAG) and cardiac surgery unit in a large geographic area $(200 \mathrm{~km})$. Outcome data, including MACE, death (cardiac vs non-cardiac), invasive angiography (IA) results (stenosis $>50 \%$ ) and coronary revascularisation procedures (either via percutaneous coronary intervention (PCI) or coronary artery bypass grafting (CABG)) were collected.

Exclusion criteria were: renal dysfunction (serum GFR $<60 \mathrm{~mL} / \mathrm{min} / 1.73 \mathrm{~m}^{2}$ ), hyperthyroidism, iodine allergy, pregnancy, known $\mathrm{CAD}$, previous PCI or CABG or previous myocardial infarction/acute coronary syndrome.

\section{Multislice CT}

From December 2005 until December 2009, a 64-slice CT (Somatom Sensation 64, Siemens, detector collimation $64 \times 0.75 \mathrm{~mm}$, rotation time $0.33 \mathrm{~s}$ ) was utilised. From December 2009, CTA exams were performed using a 128-slice dual source CT (DSCT) (Somatom Definition Flash, Siemens).

First, a non-contrast-enhanced CCS standardised CT scan (detector collimation $64 \times 1.5 \mathrm{~mm}, 120 \mathrm{kV}$, ECG-gating, slice thickness $3 \mathrm{~mm}$ and heart view medium smooth kernel B $35 \mathrm{f}$ ) was performed and the Agatston Score was calculated. ${ }^{20}$

Second, CCTA was performed. Scan parameters for 128-DSCT (Somatom Definition Flash, Siemens) and 64-slice CT (Somatom Sensation 64, Siemens) were: detector collimation $2 \times 64 \times 0.6 \mathrm{~mm}$ with a $\mathrm{z}$-flying spot and $64 \times 0.6 \mathrm{~mm}$, rotation time 0.28 and $0.33 \mathrm{~s}$, respectively. Patients were scanned in supine position in midinspiration breath-hold while an ECG was recorded.

Based on the patient's individual heart rate (HR) and body mass index (BMI), different scan protocols were used. For 128-DSCT, prospective ECG-triggering was applied for regular HR $<65 \mathrm{bpm}$ (either high-pitch FLASH-mode (pitch 3.4) if HR was $<57 \mathrm{bpm}$ and BMI $<24$ or sequential mode in the remaining) and retrospective ECG-gating was used for patients with HR $>65 \mathrm{bpm}$ or irregular HR. For 64-slice CT, retrospective ECG-gating was applied.

Tube voltage was $100 \mathrm{kV}$ in patients with $\mathrm{BMI}<26 \mathrm{~kg} / \mathrm{m}^{3}$ and $120 \mathrm{kV}$ in $\mathrm{BMI}>26 \mathrm{~kg} / \mathrm{m}^{2}$.

An iodine contrast agent (CA) with $370 \mathrm{mg} / \mathrm{mL}$ iodine concentration (Iopromide, Ultravist 370, Bayer Schering Pharma, Berlin, Germany) was triggered into arterial phase, applying bolus tracking technique, while initiating the CT scan at a threshold of 100 Hounsfield units (HU). The CA volume ranged between 65 and $120 \mathrm{cc}$ depending on the individual patient's body weight, iodine delivery rate and scan time using a standardised scheme. The CA was injected intravenously at a flow of $4-6 \mathrm{~mL} / \mathrm{s}$ followed by a $40 \mathrm{cc}$ saline solution bolus using an automated injector. 


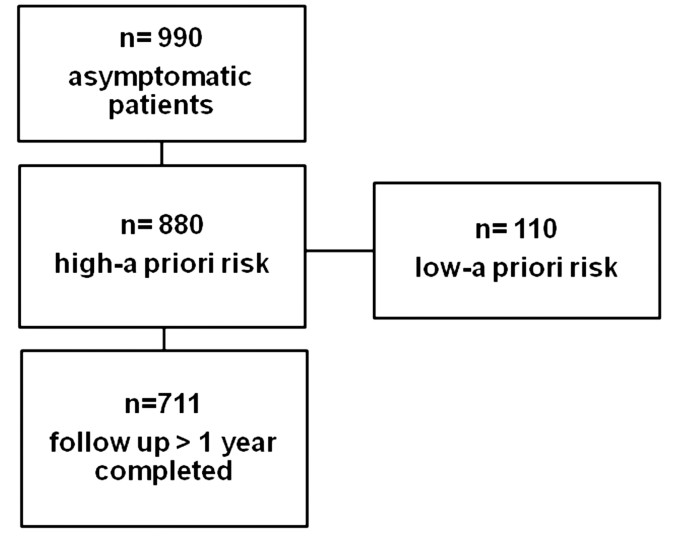

Figure 1 Patient recruitment. Out of all 990 asymptomatic patients referred to coronary CT angiography, those with high 'a priori' risk were identified. Out of those, 711 could be followed up for a minimum of 1 year (up to 8 years).

Axial images were reconstructed with $0.75 \mathrm{~mm}$ slice width (increment 0.4 ), a medium-smooth reconstruction (B $26 \mathrm{f}$ ) and evaluated using multiplanar reformation for presence of stenosis less than $50 \%, 50-70 \%$ stenosis or greater than $70 \%$. The presence and quality of coronary plaques (non-calcified, mixed or calcified) were assessed for each coronary segment by one experienced observer (10 years of training, equivalent to ACCF/AHA level 3 accreditation) on a per segment basis (AHA modified 16-segment classification). ${ }^{21}$

\section{Coronary atherosclerosis: per-segment-based plaque involvement}

The segment involvement score (SIS) was used as a quantifying measure of coronary plaque. Each segment was scored individually as 0 or 1 , based on the presence of plaques, irrespective of the degree of stenosis. The sum of all involved segments (ranging from 0 to 15) was calculated for each patient. ${ }^{22}$
Similarly, a non-calcified segment involvement score (ncSIS) was calculated. Only non-calcified hypodense plaques $^{23}$ without any calcification $(\mathrm{HU}<130)$ were included, and all involved segments summed.

\section{Outcome analysis}

Primary end point was any MACE including ST-elevation MI (STEMI), non-ST-elevation MI (NSTEMI) acute coronary syndrome or cardiac death. Non-cardiac deaths were not defined as MACE.

Secondary end points were defined as (1) coronary revascularisation rate (PCI or CABG) and (2) coronary stenosis $>50 \%$ by IA.

\section{Statistical analysis}

Statistical analysis was performed using SPSS software (V.17.0, SPSS Inc, Chicago, USA) and MedCalc (V.12.5, MedCalc Software bvba, Belgium). Quantitative variables are expressed as means $\pm \mathrm{SD}$ and categorical variables are presented as absolute values and percentages. A $p$ value of less than 0.05 was considered statistically significant. Group comparisons were performed using $\chi^{2}$ test or Fisher's exact test for categorical variables.

Kaplan-Meier log-ranked survival probability analysis was applied for the different CTA groups (stenosis severity) for primary and secondary end points. Receiver operating curve (ROC) analysis (C-index) was performed for CCS and CTA compared with stenosis $>50 \%$ in IA. ROC pairwise comparison using DeLong's method was applied to test for differences among the predictive value of CCS and CTA stenosis $>50 \%$.

The risk for adverse outcome was calculated for SIS and ncSIS scores using Cox proportional hazard risk model (DeLong's approach), and thresholds were tested stepwise with increments of 1 until a statistically significant level was reached.

\begin{tabular}{|c|c|c|c|c|c|}
\hline & $\begin{array}{l}\text { CT negative } \\
\mathrm{n}=201\end{array}$ & $\begin{array}{l}\text { CT CAD+ } \\
\text { (no stenosis) } \\
n=111\end{array}$ & $\begin{array}{l}\text { CT CAD+ } \\
<50 \% \\
n=170\end{array}$ & $\begin{array}{l}\text { CT CAD+ } \\
50-70 \% \\
n=76\end{array}$ & $\begin{array}{l}\text { CT CAD+ } \\
>70 \% \\
n=153\end{array}$ \\
\hline Age (years) & 54.6 & 62.8 & 64.5 & 65.2 & 63.5 \\
\hline Gender $(\mathrm{m} / \mathrm{f})$ & $121(60)$ & $41(37)$ & $68(40)$ & $26(34)$ & $33(22)$ \\
\hline BMI $\left(\mathrm{kg} / \mathrm{m}^{2}\right)$ & 24.5 & 25.9 & 26.5 & 26.6 & 27.2 \\
\hline \multicolumn{6}{|l|}{ Risk factors (\%) } \\
\hline Smoking & $55(34)$ & $34(35)$ & $36(26)$ & $16(27)$ & $56(42)$ \\
\hline $\mathrm{HT}$ & $70(42)$ & $47(48)$ & $82(58)$ & $40(66)$ & $93(69)$ \\
\hline $\mathrm{FH}$ & $74(46)$ & $36(40)$ & $70(51)$ & $26(43)$ & $51(39)$ \\
\hline Dyslipidaemia & $76(48)$ & $53(60)$ & $73(53)$ & $36(62)$ & $89(70)$ \\
\hline DM & $9(6)$ & $10(13)$ & $16(13)$ & $6(12)$ & 21 (19) \\
\hline Framingham Score & $6.1 \%$ & $11.3 \%$ & $11.3 \%$ & $12.1 \%$ & $14.5 \%$ \\
\hline
\end{tabular}

The patient profile is shown for each CT group.

Data expressed in numbers and percentage.

BMI, body mass index; CAD, coronary artery disease; DM, diabetes mellitus; $\mathrm{FH}$, family history, $\mathrm{HT}$, arterial hypertension. 

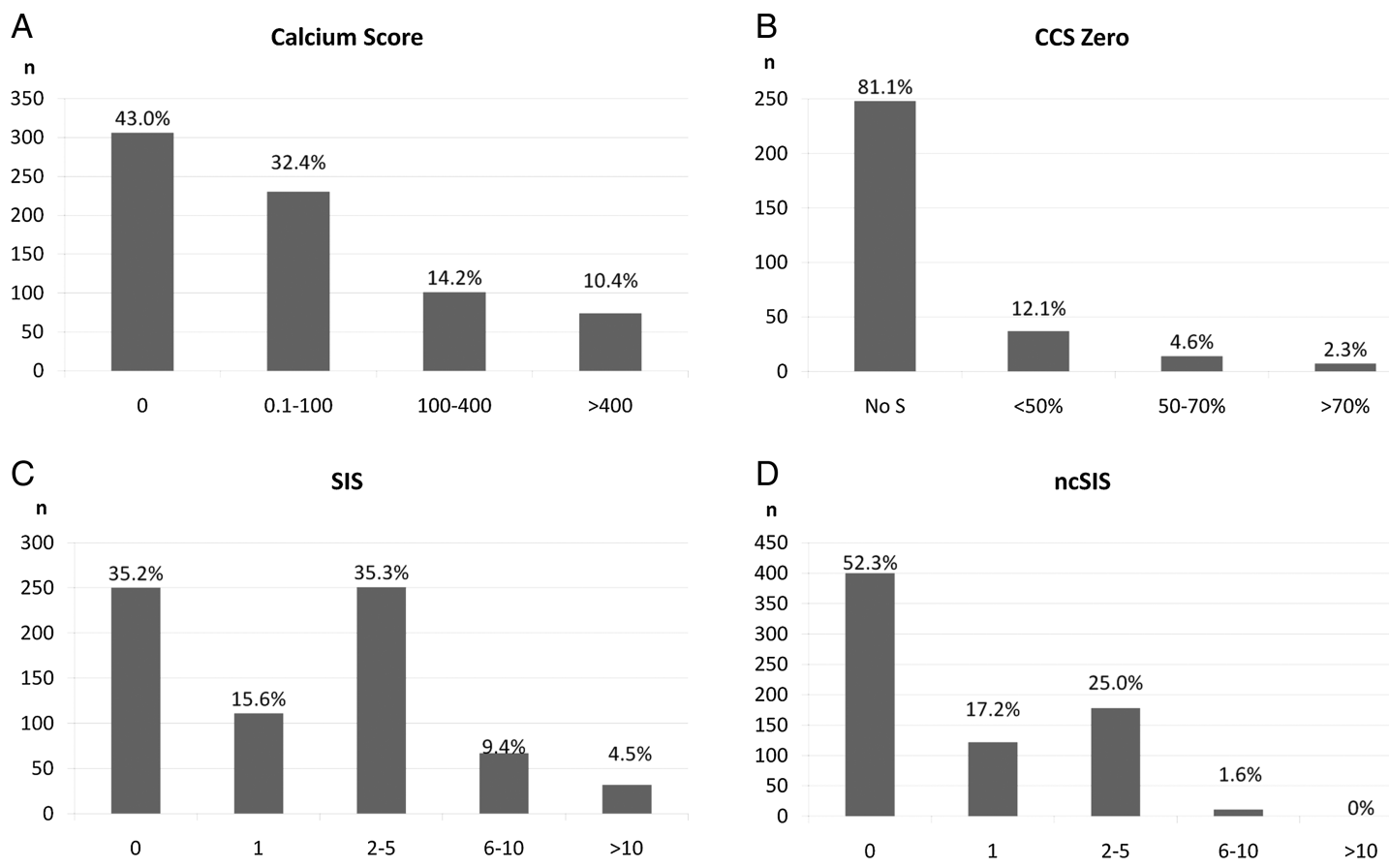

Figure 2 CT angiography results (vertical axis, $\mathrm{N}=$ count of patients): (A) total Coronary Calcium Score (CCS) (y-axis, Agatston Score), (B) coronary stenosis severity degree (\%; y-axis) in CCS zero patients (S=stenosis). (C) coronary artery plaque segment involvement score (SIS) (y-axis) and (D) non-calcified plaque SIS (ncSIS) (y-axis) shows a declining \% of patients with increasing scores.

\section{RESULTS}

Of 880 asymptomatic patients referred to coronary CT who met the inclusion criteria, $711(80.8 \%)$ could be followed up (F/U) and were finally enrolled (figure 1). $\mathrm{F} / \mathrm{U}$ period was mean 2.65 years \pm 1.5 (maximum 8 years). Table 1 shows our study cohort characteristics.

Figure 2 A illustrates CCS severity distribution. CCS zero prevalence was 306 (43\%). Out of those, noncalcified plaques were found in 98 (32\%; total prevalence of non-calcifying plaques and CCS zero, $7.6 \%)$.

While the majority of CCS zero patients (figure 2B) had no coronary stenosis $(81 \%), 37(12.1 \%)$ had $<50 \%$, $14(4.6 \%)$ had intermediate, and $7(2.3 \%)$ had high grade stenosis by CTA.

Overall, $29(19 \%)$ of 153 high-grade stenoses were caused by non-calcified plaques. Non-calcified plaques caused high-grade coronary stenosis in 15/46 (32.6\%) patients with low CCS of $\leq 100$ Agatston Units.
The distribution of total and non-calcifying plaque SIS severity stratified in groups is demonstrated in figure 2B, C. Atherosclerotic plaques were calcified $(61.7 \%)$, mixed $(20.4 \%)$ or non-calcified $(17.9 \%)$.

Figure 3 shows CTA findings and the corresponding assignment of patients into stenosis severity groups: $71.7 \%$ had signs of $\mathrm{CAD}+$ and $28.3 \%$ were CAD(15.6\% plaques without stenosis, $23.9 \%$ mild, $10.7 \%$ intermediate and $21.5 \%$ high-grade stenosis).

\section{Primary end point: MACE}

Total MACE rate was $0.8 \%(6 / 711,4$ STEMI and 2 NSTEMI; including 2 cardiac deaths after STEMI). Figure 3 shows the MACE rates among the CTA stenosis severity groups. There was no MACE in the negative CTA $(0 \%)$ group. Of six events in the CAD+ group, three patients had high-grade stenosis, two had
Figure 3 MACE rate among CAD severity groups (total $0.8 \%$; 6/711) based on CTA findings (stenosis severity). MACE occurred only in CAD-positive patients but in none $(0 \%)$ of CAD negatives by CTA CAD, coronary artery disease; CTA, CT angiography; MACE, major adverse cardiac events; $\mathrm{S}$, stenosis.

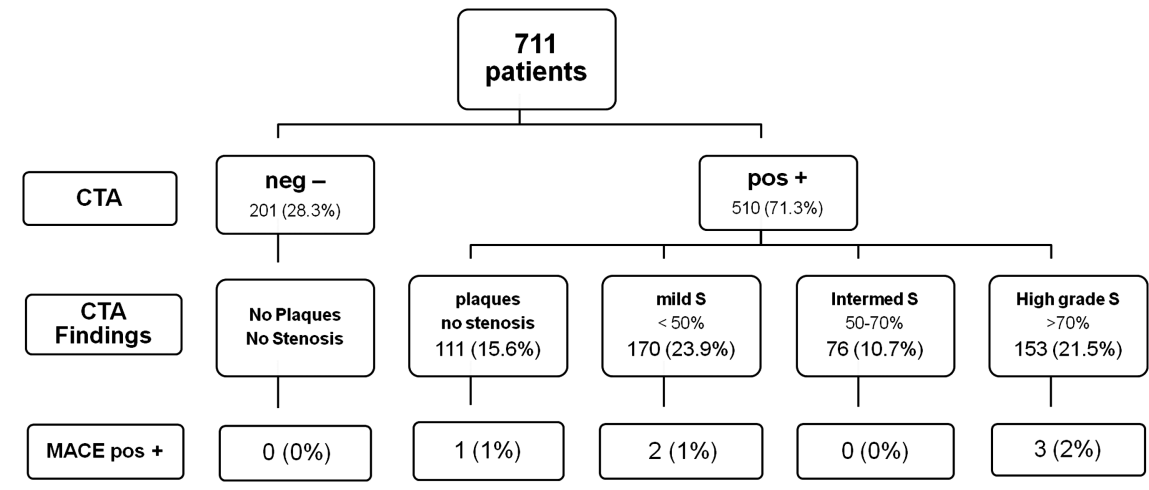




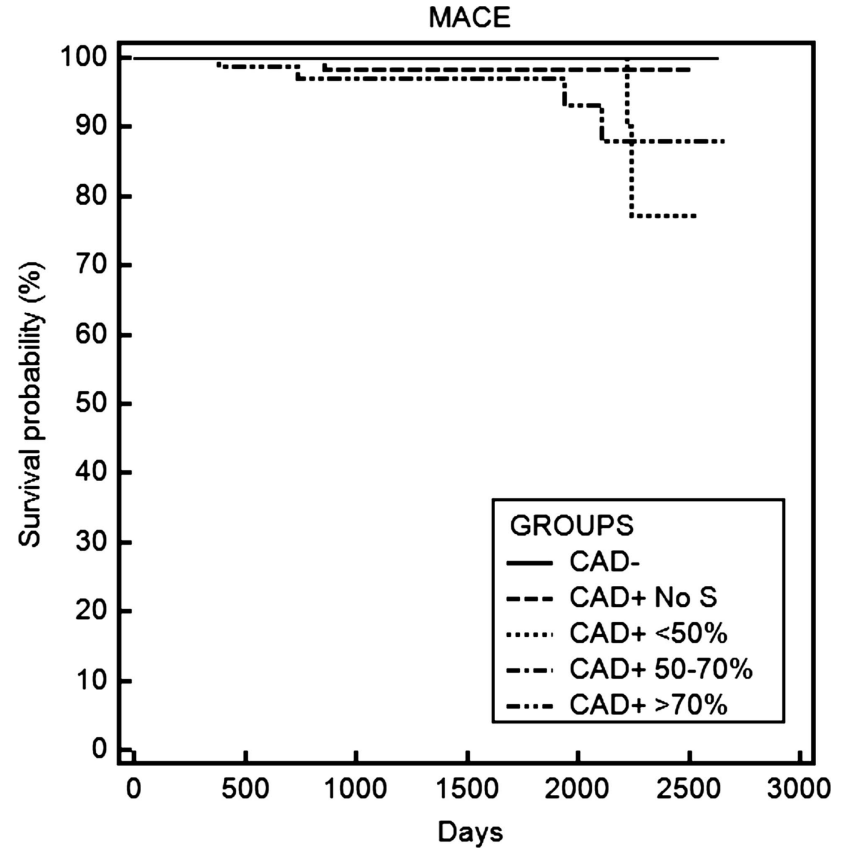

Figure 4 Kaplan-Meier survival curve for major adverse cardiac events (MACE): a trend towards superior survival in patients without signs of coronary artery disease (CAD; 0\%) as compared to CAD+ groups; with a close-to-borderline statistical significance level $(p=0.063)$, and declining survival probability with increasing stenosis severity.

intermediate and one had mild stenosis by CTA (four of six patients had non-calcifying plaque).

MACE rate in the $\mathrm{CAD}+$ group was slightly higher $(1.2 \%)$ than in the CAD- $(0 \%)$ group $(\mathrm{p}=0.27$; Fisher's exact test). The Kaplan-Meier curve for MACE (figure 4) illustrates a trend towards superior survival in CADpatients as compared with CAD+ $(\mathrm{p}=0.063)$.

Non-cardiac death was observed in five patients with metastatic disease: one pulmonary embolism/carcinoma, one sarcoma, one Merkel-cell-carcinoma and two metastatic adenocarcinoma.

\section{Secondary end points: (1) Coronary revascularisation and (2) IA $>\mathbf{5 0 \%}$ stenosis}

Total coronary revascularisation rate was $5.5 \%$ (39/711, $29 \mathrm{PCI}$ and 10 CABG surgery). Figure 5 illustrates the revascularisation rates among the different CTA stenosis severity groups, and figure 6 Kaplan-Meier survival curve.

Based on CTA findings and downstream testing, 161 patients $(22.6 \%)$ were referred to IA. Of those, $93 \mathrm{had}$ high-grade stenosis by CT, the remaining had intermediate or mild stenosis, or IA was performed due to NSTEMI or STEMI.

Compared to IA, the accuracy of CTA for the detection of coronary artery stenosis $>50 \%$ in 165 patients was: sensitivity $92.5 \% \quad(95 \%$ CI $81.8 \%$ to $97.9 \%)$. Figure 7 shows an asymptomatic patient with a $99 \%$ stenosis by CTA and IA.

For exclusion of combined primary and secondary end points, the negative predictive value (NPV) of CTA ( $>50 \%$ stenosis) compared to ICA $>50 \%$ was $99.3 \%$ (95\% CI $98.3 \%$ to $99.7 \%)$ in 711 patients.

CCS had a lower predictive value ( $=0.64 ; 95 \%$ CI 0.558 to 0.711$)$ as CTA $>50 \%$ ( $=0.71 ; 95 \%$ CI 0.632 to $0.777 ; \mathrm{p}<0.001)$ to predict $>50 \%$ stenosis by CAG $(\mathrm{p}=0.11)$.

\section{Atherosclerosis severity scores by CTA for risk prediction}

Total coronary atherosclerosis SIS was a significant predictor for MACE if five or more segments were involved (SIS $\geq 5$, hazard risk, HR 6.5; table 2) and for secondary end point if $\mathrm{SIS} \geq 1$.

ncSIS $\geq 1$ was associated with a significantly increased HR of 2.4 in patients with one or more segments with non-calcified plaques for secondary end point.

\section{DISCUSSION}

Our prospective outcome study clearly supports the use of CTA for screening of asymptomatic patients with high 'a priori' risk of CAD after conventional cardiological baseline assessment, due to the following findings: First, the prevalence of CAD in our high-risk study cohort was high with $71 \%$, which is markedly higher than in asymptomatic healthy South Koreans ${ }^{9}{ }^{10}$ with no-risk or low-risk of CAD (22\% rate for coronary stenosis of any severity by $\mathrm{CTA}^{9}$ and $11.4 \%$ atherosclerosis prevalence by CTA). Beyond ethnic and genetic differences between Asian and Caucasians, ${ }^{24}$ a higher CAD rate in
Figure 5 Coronary revascularisation rate (total $5.5 \%$; $39 / 711$ ) was $0 \%$ in patients with negative CAD findings. CAD, coronary artery disease; $\mathrm{CAG}$, coronary angiography; CTA+, positive for CAD (presence of non-calcified or calcified plaque); CTA-, negative for CAD; Revasc, revascularisation (percutaneous coronary intervention or coronary artery bypass grafting); $\mathrm{S}$, stenosis; Data are presented as $\mathrm{n}(\%)$.

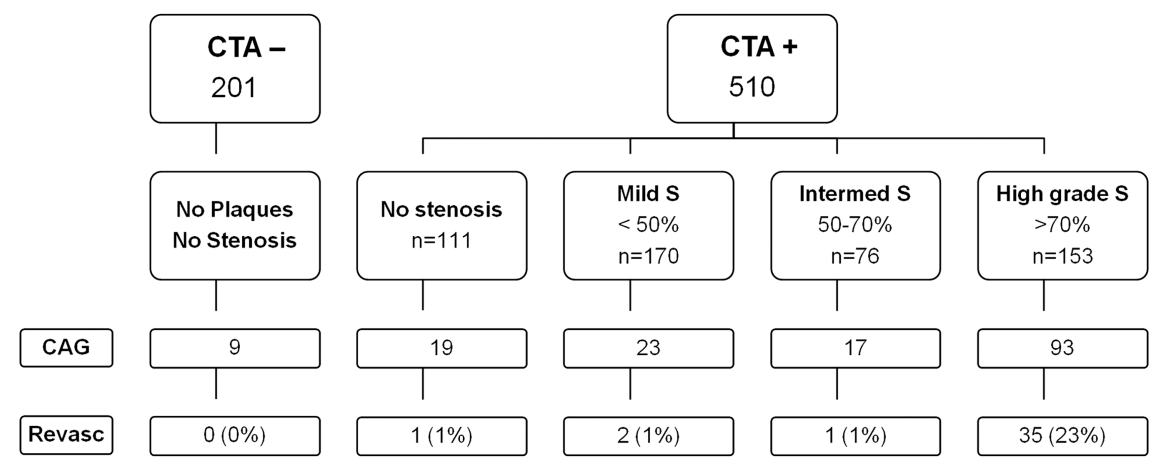




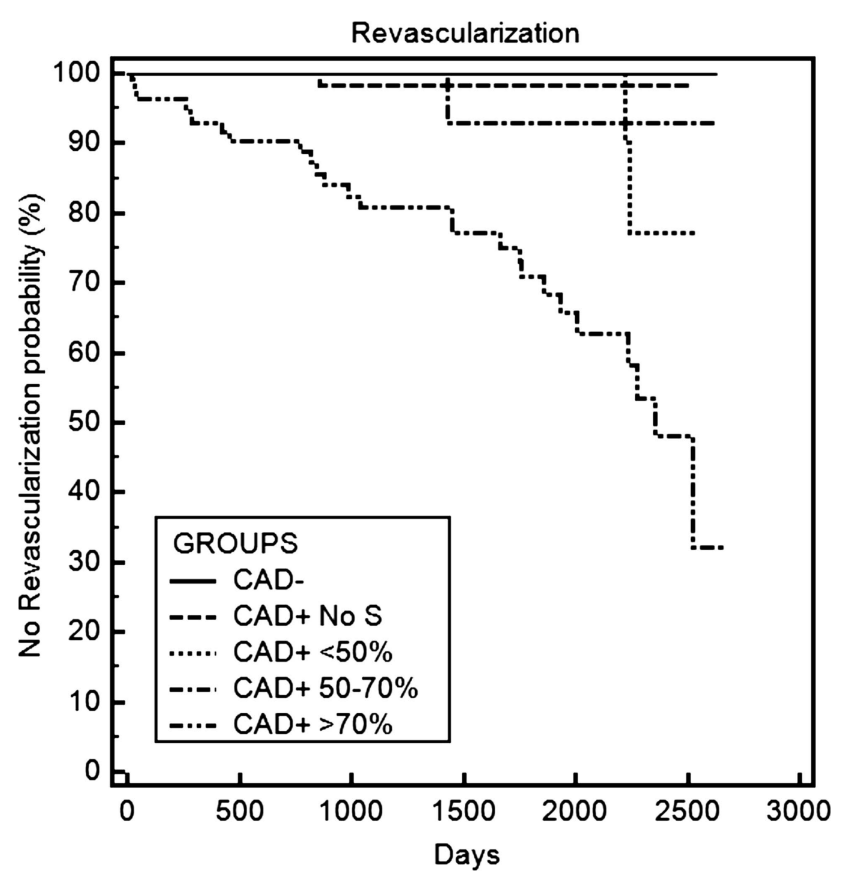

Figure 6 Kaplan-Meier curves: revascularisation rate $(\mathrm{PCl}$ or $C A B G)$ was significantly higher $(p<0.0001)$ in the CAD+ group (without a statistical difference among stenosis severity groups). CAD+, positive for coronary artery disease; $\mathrm{PCl}$, percutaneous coronary intervention; CABG, coronary artery bypass grafting.

our study is explained by the higher 'a priori' likelihood of CAD in our 'high-risk' population, which is characterised by a higher mean age, a higher rate of male gender, higher risk profile, inclusion of patients with diabetes and abnormal previous ECG-treadmill stress test. An underestimation of occult CAD using national cholesterol education programme (NCEP) guidelines as compared to CTA was suggested by Kim et al. ${ }^{10}$

In our cohort, the majority of patients had mild or intermediate stenosis. All those patients benefit from medical treatment and restrictive coronary risk control management in order to reduce progression of total atherosclerotic plaque burden. ${ }^{25}$ However, high-grade stenoses were found in one-quarter, and those patients require further diagnostic work-up, potentially including an invasive coronary angiography.

Studies investigating CTA in asymptomatic patients are rare. ${ }^{9-12}$ Beyond the two South Korean reports, only two other pivotal studies ${ }^{11}{ }^{12}$ in small cohorts showed, for example, an excellent outcome in 100 patients with normal CTA scans $(0 \%$ event rate), but a low event rate $(8 \%)$ in those with non-obstructive CAD. ${ }^{12}$

Second, our study revealed that zero CCS does not exclude CAD. CCS zero is known for a high NPV for exclusion of CAD and recommended for screening of asymptomatic patients. ${ }^{26} 27$ This finding is in line with Cheng $e t a l^{28}$ in which CCS zero failed to rule out CAD and even high-grade stenosis in selected patients due to non-calcifying plaques. Similarly, well over one-third of all plaques were non-calcified in another study. ${ }^{10}$

Our study supports the use of CTA instead of CCS for screening of asymptomatic patients for CAD by CTA, particularly in those having a high 'a priori' risk of CAD, who have a markedly higher prevalence of CAD, in contrast to no-risk or low-risk patients (eg, CCS zero rate was $93 \%$ in asymptomatic healthy Koreans). ${ }^{10}$

The advantage of CTA over CCS was highlighted in a recent data release from the CONFIRM registry, in which symptomatic patients were enrolled, which showed the incremental value of CCTA using plaque burden and stenosis severity score over CCS for discrimination of individuals at risk of death or myocardial infarct. ${ }^{29}$ Most recently, incremental value data of CTA over CCS were also released from the CONFIRM registry for 27125 asymptomatic individuals with diabetes, ${ }^{30}$ with results similar to those found in our population. Beyond age, gender and CCS (C-index 0.64), CCTA improved discrimination by maximal stenosis, number of obstructive vessels and the coronary segment stenosis score (C-index 0.77-78, respectively) in this study. ${ }^{30}$

Moreover, our data are in line with other studies emphasising no absolute linearity of increasing CCS and total coronary plaque burden. Particularly low CCS was
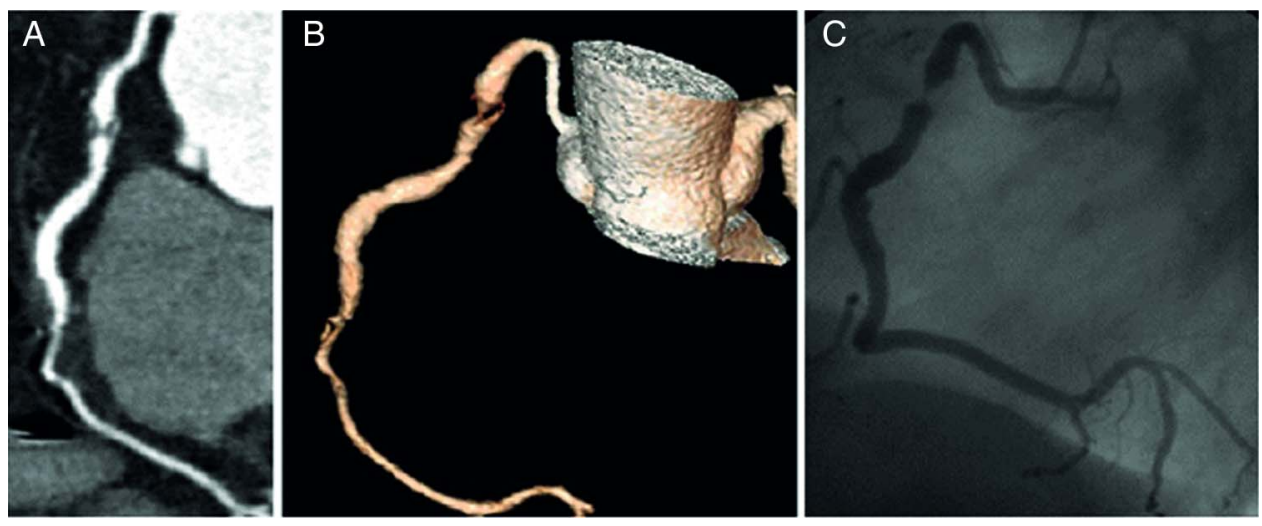

Figure 7 Asymptomatic 67-year-old man prior to orthopaedic surgery with three cardiac risk factors and borderline ST-segment depression inferior-posterior on treadmill test, caused by $99 \%$ stenosis of the right coronary artery (white arrows, A-C) and concentric non-calcified plaque (inlay right, $B$ ) which were correctly identified by $C T(A, B)$ and confirmed by invasive angiography $(C)$. 
Table 2 Cox proportional hazard risk model for primary end point (MACE) and secondary end point (revascularisation rate)

\begin{tabular}{|c|c|c|c|c|}
\hline & $\begin{array}{l}\text { MACE } \\
\text { HR }(95 \% \mathrm{CI})\end{array}$ & p Value & $\begin{array}{l}\text { Coronary revascularisation } \\
\text { HR }(95 \% \mathrm{Cl})\end{array}$ & p Value \\
\hline Smoker & $1.41(0.29$ to 6.92$)$ & 0.67 & 1.31 (0.66 to 2.62$)$ & 0.45 \\
\hline HT & 2.91 (0.35 to 24.70$)$ & 0.33 & 1.91 (0.86 to 4.23$)$ & 0.11 \\
\hline$+\mathrm{FH}$ & - & - & 0.81 (0.39 to 1.69$)$ & 0.57 \\
\hline Cholesterol & $1.02(0.66$ to 1.40$)$ & 0.94 & 1.26 (0.54 to 2.94$)$ & 0.60 \\
\hline $\mathrm{DM}$ & $1.79(0.29$ to 11.17$)$ & 0.54 & 1.46 (0.61 to 3.50$)$ & 0.40 \\
\hline \multicolumn{5}{|l|}{ CCS } \\
\hline$\geq 10$ & 5.79 (0.70 to 47.59$)$ & 0.10 & 5.05 (2.12 to 12.05$)$ & $<0.001$ \\
\hline$\geq 100$ & 3.93 (0.89 to 17.42$)$ & 0.08 & 6.01 (3.03 to 11.92$)$ & $<0.0001$ \\
\hline$\geq 400$ & 5.57 (1.26 to 24.70$)$ & $<0.05^{\star}$ & 3.90 (1.99 to 7.65$)$ & $<0.0001$ \\
\hline \multicolumn{5}{|l|}{$\mathrm{CT}^{-}$} \\
\hline$<50 \%$ & 2.87 (0.69 to 11.93$)$ & 0.15 & 0.25 (0.06 to 1.03$)$ & 0.06 \\
\hline $50-70 \%$ & - & - & 0.32 (0.04 to 2.32$)$ & 0.26 \\
\hline$>70 \%$ & $3.40(0.85$ to 13.51$)$ & 0.08 & 27.92 (9.94 to 78.43$)$ & $<0.0001^{*}$ \\
\hline \multicolumn{5}{|l|}{ SIS } \\
\hline$\geq 1$ & $1.33(0.29$ to 6.15$)$ & $p=0.72$ & 3.65 (1.9 to 7.0$)$ & $\mathrm{p}<0.0001^{*}$ \\
\hline $\begin{array}{r}\geq 5 \\
\text { ncSIS }\end{array}$ & 6.50 (1.19 to 16.02$)$ & $p<0.05^{\star}$ & 7.13 (2.2 to 23.1$)$ & $\mathrm{p}<0.0001^{*}$ \\
\hline$\geq 1$ & $1.27(0.23$ to 5.05$)$ & $p=0.74$ & 2.36 (1.20 to 4.63$)$ & $p<0.01^{*}$ \\
\hline$\geq 5$ & 5.75 (0.69 to 47.94$)$ & $p=0.11$ & $1.82(0.43$ to 747$)$ & $p=0.42$ \\
\hline
\end{tabular}

*Statistical significance.

$+\mathrm{FH}$, positive family history; CAD, coronary artery disease; CCS, coronary calcium score; DM, diabetes mellitus; HT, arterial hypertension; ncSIS, non-calcified plaque segment involvement score; SIS, segment involvement score (reflecting total plaque burden).

significantly less reliable in predicting total plaque burden due to their association with higher overall prevalence of non-calcified plaques. This has been shown in other studies with a significant stenosis rate of nearly $10 \%^{27}$ and high prevalence of non-calcifying plaques $(83.3 \%)$ in those with low CCS $(\leq 100) .^{10}$

Overall, the total rate of non-calcifying plaques and Calcium Score zero in our study cohort was 7.6\%, similar to patients with atypical chest pain and an intermediate Framingham risk profile. ${ }^{6}$ Non-calcifying plaques may cause chest pain, for example, due to endothelial dysfunction, which occurs at early stages of atherosclerosis, due to lack of regular flow-mediated vasodilation or even vasoconstriction at a lesion with mild stenosis. ${ }^{31}$

Third, our study revealed that the total and noncalcified plaque burdens (SIS and ncSIS) predict adverse outcome in terms of MACE and revascularisation procedures such as PCI or CABG, respectively, which is in line with results from the CONFIRM trial. ${ }^{32}$ In contrast, the CONFIRM trial mainly enrolled symptomatic patients.

As a study novelty, we calculated a dedicated noncalcifying plaque score (the 'ncSIS'), which showed an increased risk of adverse outcome for secondary end point, even if only one (or more) segment was involved.

An increasing total coronary plaque burden by CTA was associated with a significantly increased risk of MACE and revascularisation rate, going in line with results from multicentre-registry CONFIRM, in which were identified as risk factors. The total plaque burden, stenosis severity $^{32}$ or number of proximal segments with mixed or calcified plaques by CTA. ${ }^{33}$
Finally, our study provides strong evidence that CTA is a highly reliable and accurate tool to exclude significant CAD and MACE events over a midterm follow-up period of mean 2.65 years (up to 8 years). No cardiac events occurred in patients with negative CTA findings.

In summary, our data support screening of asymptomatic high-risk patients with CTA. Compared to CT Calcium Score, most recently introduced low-dose CT techniques such as prospective ECG-gating allow for almost equal or just slightly higher radiation exposure of $\geq 1 \mathrm{mSv}$, however, while adding iodine CA.

Compared to CCS, CTA provides the advantage of detecting non-calcifying plaque (representing early stages of atherosclerosis), coronary plaque load assessment and stenosis quantification for risk stratification. Patients with stenosis $>50 \%$ by CTA yielded a markedly higher risk of adverse outcome than increasing CCS values.

Whether follow-up of high-risk patients by CCTA should be performed is currently an open item for discussion. There are no scientific data on CTA for monitoring of CAD progression. Follow-up intervals of a minimum of 5-7 years may be reasonable for evaluation of CAD progression. In those with intermediate stenosis (50-70\%), a myocardial perfusion stress test (eg, SPECT) should be appended in order to define the haemodynamic significance of a coronary lesion.

\section{STUDY LIMITATIONS}

First, we acknowledge a low total MACE rate. One reason is the short-term follow-up period of 2.65 years, 
beyond patient population selection criteria with a low rate of diabetes $(12.4 \%)$.

Second, our inclusion criteria for definition of our cohorts 'high-risk' of CAD involved ECG-treadmill stress testing (beyond coronary risk factors). The ECG-treadmill test has a low accuracy for detection of myocardial ischaemia.

Third, the patient's current medication (aspirin, statins) was not recorded.

\section{Conclusion}

In summary, our study recommends the use of CCTA in asymptomatic patients with a high 'a priori' risk of CAD in order to detect subclinical atherosclerosis and obstructive CAD. Non-calcified plaques were found in a high amount of asymptomatic patients, even in CCS zero patients. Non-calcified plaques pose a risk for adverse outcome, even if only small numbers of coronary segments are affected.

CTA allows for coronary risk estimation based on increasing total and non-calcifying plaque burden. CTA is a safe, accurate and reliable modality for exclusion of CAD

Given low radiation exposure of CTA below $<1 \mathrm{mSv}$ using the newest low-dose prospective ECG-gated CT techniques such as high-pitch mode or iterative reconstruction, which is equivalent to CCS scanning, CTA should be favoured over CCS in asymptomatic patients with a high 'a priori' risk of CAD.

Contributors FP: data acquisition, manuscript drafting. GF: patient selection, manuscript drafting. WD: statistical analysis, manuscript drafting. AK: statistical analysis, manuscript drafting. WJ: manuscript drafting. WMF: manuscript drafting. GF: data acquisition, patient selection, manuscript drafting.

Funding This research received no specific grant from any funding agency in the public, commercial or not-for-profit sectors.

Competing interests None.

Ethics approval Local IRB.

Provenance and peer review Not commissioned; externally peer reviewed.

Data sharing statement No additional data are available.

Open Access This is an Open Access article distributed in accordance with the Creative Commons Attribution Non Commercial (CC BY-NC 3.0) license, which permits others to distribute, remix, adapt, build upon this work noncommercially, and license their derivative works on different terms, provided the original work is properly cited and the use is non-commercial. See: http:// creativecommons.org/licenses/by-nc/3.0/

\section{REFERENCES}

1. Fleg JL, Gerstenblith $\mathrm{G}$, Zonderman $\mathrm{AB}$, et al. Prevalence and prognostic significance of exercise-induced silent myocardial ischemia detected by thallium scintigraphy and electrocardiography in asymptomatic volunteers. Circulation 1990;81:428-36.

2. Deedwania PC. Silent ischemia predicts poor outcome in high-risk healthy men. J Am Coll Cardiol 2001;38:80-3.

3. He ZX, Hedrick TD, Pratt CM. Severity of coronary artery calcification by electron beam computed tomography predicts silent myocardial ischemia. Circulation 2000;101:244-51.

4. Greenland P, Bonow RO, Brundage BH, et al. ACCF/AHA 2007 clinical expert consensus document on coronary artery calcium scoring by computed tomography in global cardiovascular risk assessment and in evaluation of patients with chest pain: a report of the American College of Cardiology Foundation Clinical Expert Consensus Task Force (ACCF/AHA Writing Committee to Update the 2000 Expert Consensus Document on Electron Beam Computed Tomography) developed in collaboration with the Society of Atherosclerosis Imaging and Prevention and the Society of Cardiovascular Computed Tomography. J Am Coll Cardiol 2007;49:378-402.

5. Taylor AJ, Cerqueira M, Hodgson JM, et al. ACCF/SCCT/ACR/AHA ASE/ASNC/ NASCI/SCAI/SCMR 2010 ACCF/SCCT/ACR/AHA/ASE/ ASNC/NASCl/SCAI/SCMR 2010 Appropriate Use Criteria for Cardiac Computed Tomography. A Report of the American College of Cardiology Foundation Appropriate Use Criteria Task Force, the Society of Cardiovascular Computed Tomography, the American College of Radiology, the American Heart Association, the American Society of Echocardiography, the American Society of Nuclear Cardiology, the North American Society for Cardiovascular Imaging, the Society for Cardiovascular Angiography and Interventions, and the Society for Cardiovascular Magnetic Resonance. Circulation 2010;122:e525-55.

6. Hausleiter J, Meyer T, Hadamitzky M, et al. Prevalence of noncalcified coronary plaques by 64 -slice computed tomography in patients with an intermediate risk for significant coronary artery disease. J Am Coll Cardiol 2006;48:312-18.

7. Hoffmann U, Moselewski F, Nieman K, et al. Noninvasive assessment of plaque morphology and composition in culprit and stable lesions in acute coronary syndrome and stable lesions in stable angina by multidetector computed tomography. J Am Coll Cardiol 2006;47:1655-62.

8. Dohi T, Mintz GS, McPherson JA. Non-fibroatheroma lesion phenotype and long-term clinical outcomes: a substudy analysis from the PROSPECT Study. JACC Cardiovasc Imaging 2013;6:908-16.

9. Choi EK, Choi SI, Rivera JJ, et al. Coronary computed tomography angiography as screening tool for the detection of occult coronary artery disease in asymptomatic individuals. J Am Coll Cardiol 2008;52:357-65.

10. Kim KJ, Choi SI, Lee MS, et al. The prevalence and characteristics of coronary atherosclerosis in asymptomatic subjects classified as low risk based on traditional risk stratification algorithm: assessment with coronary CT angiography. Heart 2013;99:1113-17.

11. Romeo F, Leo R, Clementi F, et al. Multislice computed tomography in an asymptomatic high-risk population. Am J Cardiol 2007:99:325-8.

12. Pundziute G, Schuijf JD, Jukema JW, et al. Prognostic value of multislice computed tomography coronary angiography in patients with known or suspected coronary artery disease. J Am Coll Cardiol 2007;49:62-70.

13. Hausleiter J, Meyer T, Hermann F, et al. Estimated radiation dose associated with cardiac CT angiography. JAMA 2009;301:500-7.

14. Achenbach S, Marwan M, Ropers D, et al. Coronary computed tomography angiography with a consistent dose below $1 \mathrm{mSv}$ using prospectively electrocardiogram- triggered high-pitch spiral acquisition. Eur Heart J 2010;31:340-6.

15. Schuhbaeck A, Achenbach S, Layritz C, et al. Image quality of ultralow radiation exposure coronary $\mathrm{CT}$ angiography with an effective dose $<0.1 \mathrm{mSv}$ using high-pitch spiral acquisition and raw databased iterative reconstruction. Eur Radiol 2013;23:597-606.

16. Reiner Z, Catapano AL, De Backer G, et al. ESC/EAS Guidelines for the management of dyslipidaemias: the Task Force for the management of dyslipidaemias of the European Society of Cardiology (ESC) and the European Atherosclerosis Society (EAS). Eur Heart J 2011;32:1769-818.

17. Mancia G, Fagard R, Narkiewicz K, et al. 2013 ESH/ESC guidelines for the management of arterial hypertension: the Task Force for the Management of Arterial Hypertension of the European Society of Hypertension (ESH) and of the European Society of Cardiology (ESC). Eur Heart J 2013;34:2159-219.

18. Gibbons RJ, Balady GJ, Bricker JT, et al. ACC/AHA 2002 guideline update for exercise testing: summary article. a report of the American College of Cardiology/American Heart Association Task Force on Practice Guidelines. Circulation 2002;106:1883-92.

19. Berry JD, Dyer A, Cai X, et al. Lifetime risks of cardiovascular disease. N Engl J Med 2012;366:321-9.

20. Agatston AS, Janowitz WR, Hildner FJ, et al. Quantification of coronary artery calcium using ultrafast computed tomography. J Am Coll Cardiol 1990;15:827-32.

21. Austen WG, Edwards JE, Frye RL, et al. A reporting system on patients evaluated for coronary artery disease: Report of the Ad Hoc 
Committee for Grading of Coronary Artery Disease, Council on Cardiovascular Surgery. Circulation 1975;51:5-40.

22. Øvrehus KA, Marwan M, Bøtker HE, et al. Reproducibility of coronary plaque detection and characterization using low radiation dose coronary computed tomographic angiography in patients with intermediate likelihood of coronary artery disease (ReSCAN study). Int J Cardiovasc Imaging 2012;28:889-99.

23. Leber AW, Knez A, von Ziegler F, et al. Quantification of obstructive and nonobstructive coronary lesions by 64-slice computed tomography: a comparative study with quantitative coronary angiography and intravascular ultrasound. J Am Coll Cardiol 2005;46:147-54.

24. Hulten E, Villines TC, Cheezum MK, et al. CONFIRM Investigators. Usefulness of coronary computed tomography angiography to predict mortality and myocardial infarction among Caucasian, African and East Asian ethnicities (from the CONFIRM [Coronary CT Angiography Evaluation for Clinical Outcomes: An International Multicenter] Registry). Am J Cardiol 2013;111:479-85.

25. Nissen SE, Nicholls SJ, Sipahi I, et al.; ASTEROID Investigators. Effect of very high-intensity statin therapy on regression of coronary atherosclerosis: the ASTEROID trial. JAMA 2006;295:1556-6.

26. Shareghi S, Ahmadi N, Young E, et al. Prognostic significance of zero coronary calcium scores on cardiac computed tomography. J Cardiovasc Comput Tomogr 2007;1:155-9.

27. Greenland P, LaBree L, Azen SP, et al. Coronary artery calcium score combined with Framingham score for risk prediction in asymptomatic individuals. JAMA 2004;291:210-15.
28. Cheng VY, Lepor NE, Madyoon $\mathrm{H}$, et al. Presence and severity of noncalcified coronary plaque on 64-slice computed tomographic coronary angiography in patients with zero and low coronary artery calcium. Am J Cardiol 2007:99:1183-6.

29. Al-Mallah MH, Qureshi W, Lin FY, et al. Does coronary CT angiography improve risk stratification over coronary calcium scoring in symptomatic patients with suspected coronary artery disease? Results from the prospective multicenter international CONFIRM registry. Eur Heart J Cardiovasc Imaging 2014;15:267-74.

30. Min JK, Labounty TM, Gomez MJ, et al. Incremental prognostic value of coronary computed tomographic angiography over coronary artery calcium score for risk prediction of major adverse cardiac events in asymptomatic diabetic individuals. Atherosclerosis 2014;232:298-304

31. Heusch G, Baumgart D, Camici P, et al. Alpha-adrenergic coronary vasoconstriction and myocardial ischemia in humans. Circulation 2002;101:689-94.

32. Otaki $\mathrm{Y}$, Arsanjani $\mathrm{R}$, Gransar $\mathrm{H}$, et al. What have we learned from CONFIRM? Prognostic implications from a prospective multicenter international observational cohort study of consecutive patients undergoing coronary computed tomographic angiography. J NuCl Cardiol 2012;19:787-95.

33. Hadamitzky M, Achenbach S, Al-Mallah M, et al.; CONFIRM Investigators. Optimized prognostic score for coronary computed tomographic angiography: results from the CONFIRM registry (COronary CT Angiography EvaluatioN For Clinical Outcomes: an InteRnational Multicenter Registry). J Am Coll Cardiol 2013;62:468-76. 